Artigo Original

Original Article

Martha Marcela de Matos Bazilio ${ }^{1,2}$ (C) Adriana Fernandes Duarte dos Santos ${ }^{1,2}$ (1)

Silvana Frota ${ }^{1}$ (1)

Marília Guimarães ${ }^{1}$ (1)

Márcia Gonçalves Ribeiro ${ }^{1}$

Descritores

Síndrome de Turner

Disgenesia Gonadal 45X

Via Eferente

Complexo Olivar Superior

Emissões Otoacústicas

Keywords

Turner Syndrome

XO Gonadal Dysgenesis

Efferent pathways

Superior olivary complex Otoacoustic emissions

\section{Efeito inibitório da via auditiva eferente na síndrome de Turner}

\author{
Efferent Auditory Pathways Inhibition in Turner \\ syndrome
}

\section{RESUMO}

Objetivo: investigar o efeito inibitório da via auditiva eferente na síndrome de Turner e relacionar com o perfil citogenético. Método: estudo descritivo transversal com grupo de comparação. Amostra: Grupo estudo formado por 40 pacientes com síndrome de Turner (17,6 anos); e Grupo controle constituído por 54 indivíduos (18,9 anos), do sexo feminino sem síndrome. Os indivíduos selecionados foram submetidos à pesquisa do efeito inibitório da via auditiva eferente. Resultados: A média do Efeito inibitório da via auditiva eferente no grupo estudo na orelha direita foi $0,4 \mathrm{~dB}$ e no grupo comparação foi de $1,9 \mathrm{~dB}$, entretanto na orelha esquerda a média do efeito inibitório da via auditiva eferente foi $1,4 \mathrm{~dB}$ no grupo estudo e $0,8 \mathrm{~dB}$ no grupo comparação. $\mathrm{O}$ efeito inibitório da via auditiva eferente foi presente em 14 indivíduos com monossomia e em 15 com outras alterações citogenéticas. Conclusão: No grupo estudo o valor do efeito inibitório da via auditiva eferente foi significantemente maior na orelha esquerda e significativamente menor que o grupo controle na direita. Não houve diferença significativa no efeito inibitório da via auditiva eferente entre os tipos de cariótipo.

\begin{abstract}
Purpose: The goal of this study is to investigate the efferent auditory pathways inhibition in Turner's syndrome and to relate it to the cytogenetic profile. Methods: This is a cross-sectional study with a comparison group. A sample with 94 participants divided into two groups: The study group was a sample of 40 patients diagnosed with Turner's syndrome (17.6 years of age). The control group was composed of 54 volunteers (18.9 years of age), female, without syndrome. The selected individuals were submitted to efferent auditory pathways inhibition research. Results: The mean of the inhibitory effect of the efferent auditory pathway in the study group in the right ear was $0.4 \mathrm{~dB}$ and in the comparison group it was $1.9 \mathrm{~dB}$, however in the left ear the mean of the inhibitory effect of the efferent auditory pathway was $1.4 \mathrm{~dB}$ in the study group and $0.8 \mathrm{~dB}$ in the comparison group. The inhibitory effect of the efferent auditory pathway was present in 14 individuals with monosomy and in 15 with other cytogenetic alterations. Conclusions: In the study group, the efferent auditory pathways inhibition value was significantly higher in the left ear and significantly lower than the control group in the right ear. There was no significant difference in efferent auditory pathways inhibition of right ear and left ear between the karyotype types.
\end{abstract}

Endereço para correspondência: Martha Marcela de Matos Bazilio Divisão de Audiologia, Instituto Nacional de Educação de Surdos Rua das Laranjeiras, 232, Laranjeiras, Rio de Janeiro (RJ), Brasil, CEP: 22240-000.

E-mail: celambazilio@gmail.com
Trabalho realizado na Universidade Federal do Rio de Janeiro - UFRJ - Rio de Janeiro (RJ), Brasil e Instituto Nacional de Educação de Surdos - INES - Rio de Janeiro (RJ), Brasil.

${ }^{1}$ Universidade Federal do Rio de Janeiro - UFRJ - Rio de Janeiro (RJ), Brasil.

${ }^{2}$ Instituto Nacional de Educação de Surdos - INES - Rio de Janeiro (RJ), Brasil.

Fonte de financiamento: nada a declarar

Conflito de interesses: nada a declarar. 


\section{INTRODUÇÃO}

A síndrome de Turner (ST) é uma alteração cromossômica que afeta aproximadamente um em cada 2000 nascidos do sexo feminino ${ }^{(1)}$. Dentre as alterações citogenéticas relacionadas à síndrome temos classicamente a monossomia do cromossomo $\mathrm{X}$, alterações estruturais e mosaicismo que conjuga alterações numéricas e/ou estruturais com linhagem cromossômica normal, além da presença total ou parcial do cromossomo $\mathrm{Y}^{(1)}$.

O fenótipo característico da ST consta de baixa estatura proporcionada, face triangular, retrognatismo, palato ogival, pescoço curto e/ou alado, baixa implantação de cabelos na nuca, hipertelorismo mamilar, leve pectus excavatum, cúbito valgo e hipodesenvolvimento dos caracteres sexuais. Ocorrem anomalias renais e cardiovasculares, além de comprometimento da função visual e auditiva ${ }^{(2)}$. Em relação aos aspectos auditivos, até o momento, comumente são relatados diversos tipos de perda auditiva na referida síndrome, porém poucos estudos fazem a associação com os aspectos auditivos centrais ${ }^{(1 ; 3 ; 4 ; 5)}$. A Perda auditiva condutiva pode ser explicadas pela presença do palato em ogiva nos pacientes com ST, o que facilita distúrbios respiratórios, e dificulta a eliminação de secreções, podendo resultar em infecções de orelha média ${ }^{(3)}$. Outro fator causal a ser considerado são as mudanças congênitas na anatomia da tuba auditiva ${ }^{(3)}$. Além disso, pode ser observado na ST perda auditiva neurossensorial progressiva, a qual pode ser justificada pela presbiacusia precoce ocasionada pela deficiência de estrogênio nesses pacientes ${ }^{(4 ; 5)}$. Entretanto, a fisiopatologia que envolve as vias auditivas centrais na ST ainda é desconhecida ${ }^{(4)}$.

A avaliação auditiva engloba, não apenas a audição periférica, mas também as vias auditivas centrais, uma vez que existem pessoas que apresentam audição dentro dos padrões de normalidade, mas relatam queixa de não ouvir bem, principalmente em ambientes ruidosos. Desta forma, é importante o conhecimento da integridade e funcionalidade do sistema auditivo como um todo. A integridade das vias auditivas centrais aferentes e eferentes associada à ação conjunta das mesmas conduz ao funcionamento adequado do sistema auditivo central ${ }^{\left({ }^{(}\right)}$. As vias auditivas eferentes atuam na modulação das células ciliadas externas da cóclea, diminuição do potencial de ação do nervo coclear, proteção contra o ruído, localização da fonte sonora, melhora da detecção da fonte sonora em ambientes ruidosos e atenção seletiva ${ }^{(7 ; 8)}$. Estas habilidades são fundamentais para o correto processamento das informações auditivas ${ }^{(9)}$.

A integridade da via auditiva eferente, ou seja, o efeito inibitório da via auditiva eferente (EIVE) pode ser avaliado através da atenuação das Emissões Otoacústicas (EOA) na presença de ruído contralateral. Esta atenuação ocorre devido à ação do trato olivococlear medial, nas células ciliadas externas, atenuando o ganho da amplificação coclear, e consequentemente, reduzindo a movimentação da membrana basilar ${ }^{(10)}$.

Dentre as alterações relatadas na ST, podemos encontrar não apenas alterações auditivas, mas também déficits neuro funcionais ${ }^{(11)}$ associados às funções de localização sonora, percepção e atenção seletiva, funções essas atribuídas à via auditiva eferente ${ }^{(7,8)} \mathrm{O}$ conhecimento sobre a função auditiva na ST vem aumentando e até onde sabemos, o estudo da condição da via auditiva eferente na referida síndrome ainda não foi abordado. Este estudo teve como objetivo investigar o EIVE na ST e relacionar com o perfil citogenético.

\section{MÉTODO}

Foi realizado a cross-sectional study com um grupo de comparação, aprovado pelo Comitê de Ética e Pesquisa do Instituo de Puericultura e Pediatria Martagão Gesteira (IPPMG/UFRJ) e do Instituto Nacional de Educação de Surdos (INES), sob os números: 1864085, 2960593 respectivamente. Todos os indivíduos que participaram do estudo assinaram o termo de consentimento livre e esclarecido e o termo de assentimento quando necessário. As avaliações auditivas foram realizadas na Divisão de Audiologia do Instituto Nacional de Educação de Surdos (DIAU/INES) por duas fonoaudiólogas capacitadas e treinadas.

Foram estudados 94 indivíduos com idade de 9 a 39 anos, que foram divididos em dois grupos: o Grupo estudo (GE) e o Grupo Controle (GC). O GE foi formado por amostra de conveniência de base hospitalar, composta por 40 pacientes diagnosticadas com ST, média de idade 17,6 anos $( \pm 7,16)$, procedentes dos Serviços de Genética Médica e de Endocrinologia Pediátrica do IPPMG/UFRJ e do Serviço de Endocrinologia do Hospital Universitário Clementino Fraga Filho (HUCFF/UFRJ).

Os critérios de inclusão para o GE foram: diagnóstico citogenético da ST, verificado através do registro no prontuário do Laboratório de Citogenética do IPPMG e/ou do serviço de Endocrinologia do HUCFF/UFRJ. Os critérios de exclusão foram: presença de qualquer outra alteração genética, verificada através do registro no prontuário; existência de alteração cognitiva e /ou neurológica que dificultasse a compreensão para as instruções de realização dos exames, observada através da anamnese; presença de corpo estranho e/ou rolha de cera, identificado através da meatoscopia; doenças otológicas de orelha externa $(\mathrm{OE})$ e/ ou orelha média $(\mathrm{OM})$, verificada através do exame de imitanciometria; perda auditiva, de acordo com a OMS $1997^{(12)}$,ou seja, limiares inferiores a $25 \mathrm{~dB}$, na audiometria tonal; resultados no Índice de Reconhecimento de Fala (IRF) inferiores a $88 \%{ }^{(13)}$ ou ausência de respostas nas EOAT.

Já o GC foi constituído por 54 voluntárias, com média de idade 18,9 anos $( \pm 7,1)$, do sexo feminino sem ST. Os critérios de inclusão foram ser do sexo feminino em faixa etária semelhante ao do GE; os critérios de exclusão foram semelhantes ao do GE, somado ao fato de não apresentar baixa estatura, fenótipo frequente na ST.

Após a verificação dos critérios de inclusão e exclusão, duas fonoaudiólogas capacitadas e treinadas (MMMB e AFDS) aplicaram a avaliação do Efeito Inibitório da Via Eferente (EIVE), que foi avaliado através da captação e registro das EOAT com e sem estímulo acústico contralateral. Foram utilizados os seguintes parâmetros para realização das EOAT: estímulo à intensidade de $60 \mathrm{~dB}$, reprodutibilidade maior que $70 \%$, janela de análise de aproximadamente $20 \mathrm{~ms}$, faixa de frequência de $1 \mathrm{KHz}$ a $5 \mathrm{KHz}$. Foi considerada presença de resposta quando a amplitude das EOAT foi maior ou igual a $3 \mathrm{~dB}$ NPS acima do ruído, em no mínimo 3 frequências consecutivas. Este mesmo procedimento em seguida foi repetido, porém com a presença de ruído branco contralateral a $60 \mathrm{~dB}$ NPS, ou seja, tanto o estímulo como o 
ruído foram aplicados a $60 \mathrm{~dB}$ (relação sinal do estímulo e o ruído contralateral foi de $0 \mathrm{~dB}$ ) ${ }^{(14)}$. Para a captação das EOAT utilizamos o equipamento eclipse EP 25 e para o ruído utilizamos o audiômetro Ad 229-b, devidamente calibrados.

O EIVE foi determinado pela subtração do nível de resposta das EOAT sem ruído branco contralateral captado em um primeiro momento (M1) do nível de resposta das EAOT com ruído branco contralateral, captadas em um segundo momento (M2). A fórmula encontra-se a seguir ${ }^{(15)}$.

1.

EIVE = EOAT sem ruído (M1) - EOAT com ruído (M2)

Fonte: Modificado de Collet, $1992^{(15)}$

Foram consideradas respostas positivas (presença do EIVE) quando houve redução maior ou igual a $1 \mathrm{~dB}$ entre amplitude de respostas das emissões otoacústicas sem e com presença de ruído branco contralateral, indicando normalidade da via auditiva eferente. Considerou-se ausência do EIVE, quando a redução da amplitude for inferior à $1 \mathrm{~dB}$, considerando a resposta das emissões com e sem presença de ruído branco contralateral (ausência do EIVE) ${ }^{(15)}$.

Após terem sido determinados os resultados do EIVE dos participantes de cada grupo, foram comparados os valores encontrados no GE com o GC. A variação entre os momentos M1 e M2 no interior de cada grupo foi avaliada pelo teste dos postos sinalizados de Wilcoxon e a comparação das medidas entre os dois grupos pelo teste de Mann-Whitney. A comparação entre os dados categóricos (presença/ausência do EIVE) entre os grupos foi analisada pelo teste de qui-quadrado $\left(\chi^{2}\right)$.

Os cariótipos encontrados nos prontuários, foram divididos em dois tipos: a) o Tipo 1 (T1): monossomia do cromossomo X e b) Tipo 2 (T2): outras alterações citogenéticas. Sendo assim, verificamos a relação entre o resultado do EIVE (presente/ausente) entre os dois tipos de cariótipo (T1 e T2), que foi analisada pelo teste exato de Fisher. Foi considerada análise por indivíduo e não por orelha; para tal o EIVE foi considerado presente quando pelo menos uma das orelhas apresentou resultado positivo.
A análise descritiva apresentou os dados observados na forma de tabelas e gráficos ilustrativos. Os dados numéricos foram expressos pelas medidas de tendência central e de dispersão adequados, e os dados categóricos pela frequência e porcentagem.

Além disso, foi realizada uma análise prévia para verificar a normalidade das variáveis. Para tal, foi utilizado o teste de ShapiroWilk juntamente com a análise gráfica dos histogramas. Uma vez que as variáveis apresentaram distribuição não-Gaussiana, em pelo menos um momento e/ou grupo, foram utilizados testes não paramétricos. Sendo assim, as medidas mais adequadas para sumarização dos dados são pelos quartis (mediana e intervalo interquatílico (Q1-Q3). O critério de determinação de significância adotado foi o nível de 5\%. A análise estatística foi processada pelo software estatístico SPSS versão 26.

\section{RESULTADOS}

Inicialmente foram atendidas 88 pacientes com ST, sendo que 23 foram excluídas por apresentarem alteração cognitiva e/ou neurológica que dificultou a compreensão das tarefas propostas, uma por apresentar outra síndrome associada e 24 por apresentarem alteração auditiva no sistema auditivo periférico, sendo elegíveis 40 indivíduos para o estudo. Já para o GC, foram avaliados 54 indivíduos do sexo feminino e todas atenderam os critérios de inclusão estabelecidos e nenhuma foi excluída.

A Tabela 1 apresenta média, mediana e o intervalo interquartílico (IIQ: Q1 - Q3) das medidas da OD e OE nos momentos M1 e M2 segundo os grupos GE e GC.

No grupo estudo, foi observado variação (queda) significativa do M1 para M2 na OE ( $p$-valor $=0,014$ ) e no grupo controle foi observado queda significativa do M1 para M2 na OD ( $p$-valor = $0,001)$. Além disso, verificou-se que o grupo estudo apresentou nível de resposta do EIVE significantemente menor na OD que o grupo controle ( $p$-valor $=0,018$ ).

A Figura 1 ilustra as medianas da OD e da OE nos momentos M1 e M2 segundo os grupos GE e GC.

Na Tabela 2 é possível ver o número (n) e porcentagem (\%) do EIVE da OD e OE segundo os grupos GE e GC.

Tabela 1. Medidas descritivas das EOAT (OD e OE) nos momentos M1 e M2 com os respectivos EIVE segundo os grupos GE e GC

\begin{tabular}{|c|c|c|c|c|c|c|c|}
\hline \multirow{2}{*}{ Variável } & \multicolumn{3}{|c|}{ Grupo Estudo $(n=40)$} & \multicolumn{3}{|c|}{ Grupo Controle $(n=54)$} & \multirow{2}{*}{$p$ valor ${ }^{2}$} \\
\hline & média & mediana & $\| \mathrm{Q}$ & média & mediana & IIQ & \\
\hline \multicolumn{8}{|c|}{ Orelha Direita } \\
\hline M2 & 17,8 & 19,0 & $15,0-21,8$ & 16,4 & 16,5 & $12,8-21,3$ & 0,24 \\
\hline$p$ valor ${ }^{1}$ & \multicolumn{3}{|c|}{0,87} & \multicolumn{4}{|c|}{0,001} \\
\hline EIVE & 0,4 & 0 & $-1,8-1,0$ & 1,9 & 1,0 & $-0,3-4,0$ & 0,018 \\
\hline M1 & 18,4 & 18,0 & $16,3-21,8$ & 17,9 & 19,0 & $13,0-22,3$ & 0,90 \\
\hline M2 & 17,0 & 18,0 & $16,0-21,0$ & 17,1 & 18,0 & $11,8-22,0$ & 0,95 \\
\hline$p$ valor 1 & \multicolumn{3}{|c|}{0,014} & \multicolumn{4}{|c|}{0,089} \\
\hline EIVE & 1,4 & 1,0 & $-0,8-2,8$ & 0,8 & 1,0 & $-1,0-3,0$ & 0,61 \\
\hline
\end{tabular}

Os dados foram expressos pela média, mediana, e intervalo interquartílico IIQ: Q1-Q3. ${ }^{1} \mathrm{~A}$ comparação entre os momentos M1 e M2 foi avaliada pelo teste dos postos sinalizados de Wilcoxon; ${ }^{2} \mathrm{~A}$ comparação entre grupos (estudo e controle) foi avaliada pelo teste de Mann-Whitney

Legenda: $\mathrm{M} 1$ = momento $1 ; \mathrm{M} 2$ = momento 2; IIQ = intervalo interquartílico; EIVE = Efeito Inibitório da via auditiva eferente 


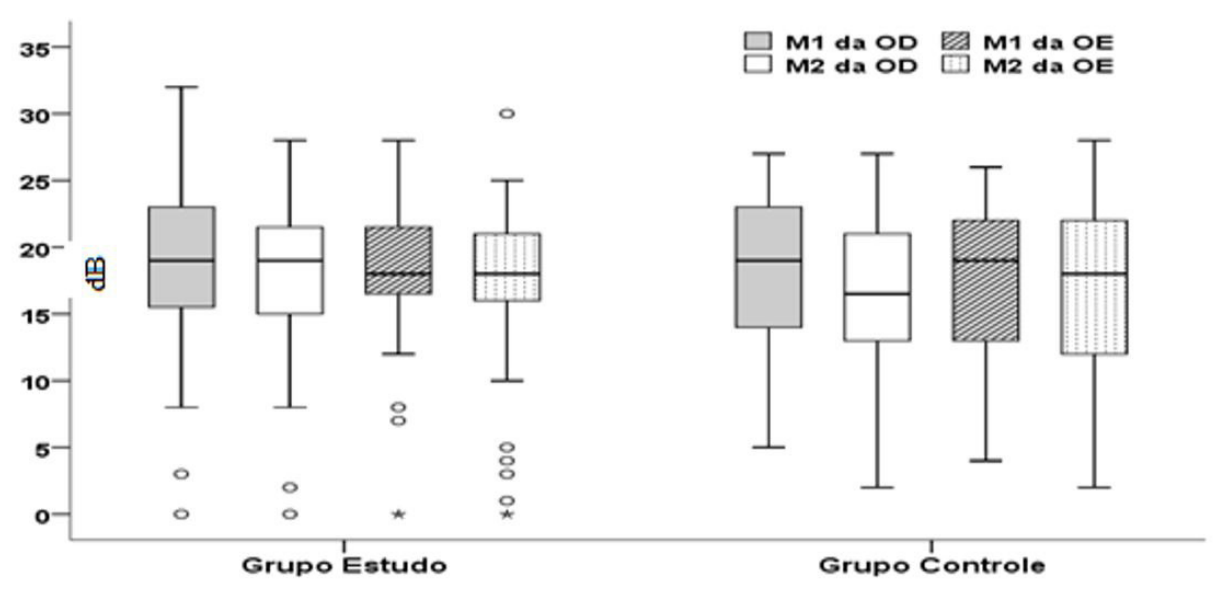

M1 = momento 1; M2 = momento 2; OD = orelha direita; OE = orelha esquerda; GE = grupo estudo; GC = grupo controle; EOAT = Emissões Otoacústicas Transientes Figura 1. Medianas das EOAT das orelhas (OD e OE) nos momentos M1 e M2 (grupos GE e GC)

Tabela 2. Distribuição do EIVE segundo os grupos GE e GC

\begin{tabular}{|c|c|c|c|c|c|}
\hline \multirow{2}{*}{ EIVE } & \multicolumn{2}{|c|}{ Grupo Estudo } & \multicolumn{2}{|c|}{ Grupo Controle } & \multirow{2}{*}{$p$ valor } \\
\hline & $\mathrm{n}$ & $\%$ & $\mathrm{n}$ & $\%$ & \\
\hline \multicolumn{6}{|c|}{ Orelha Direita } \\
\hline presente & 14 & 35,0 & 31 & 57,4 & \multirow{2}{*}{0,032} \\
\hline ausente & 26 & 65,0 & 23 & 42,6 & \\
\hline \multicolumn{6}{|c|}{ Orelha Esquerda } \\
\hline presente & 25 & 62,5 & 29 & 53,7 & \multirow{2}{*}{0,39} \\
\hline ausente & 15 & 37,5 & 25 & 46,3 & \\
\hline
\end{tabular}

Os dados foram expressos pela frequência $(n)$ e porcentagem (\%) e comparados pelo teste $\chi 2$

Legenda: EIVE = Efeito Inibitório da via auditiva eferente

Tabela 3. Distribuição do EIVE segundo os tipos de cariótipo (T1 e T2)

\begin{tabular}{|c|c|c|c|c|c|}
\hline \multirow{2}{*}{ EIVE } & \multicolumn{2}{|c|}{ Presente } & \multicolumn{2}{|c|}{ Ausente } & \multirow{2}{*}{$p$ valo } \\
\hline & $\mathrm{n}$ & $\%$ & $\mathrm{n}$ & $\%$ & \\
\hline \multicolumn{6}{|c|}{ Cariótipo } \\
\hline $\mathrm{T} 1$ & 14 & $48,3 \%$ & 3 & $27,3 \%$ & \multirow{2}{*}{0,20} \\
\hline T2 & 15 & $51,7 \%$ & 8 & $72,7 \%$ & \\
\hline
\end{tabular}

Os dados foram expressos pela frequência (n) e porcentagem (\%) e comparados pelo teste de Teste exato de Fisher

Legenda: EIVE = Efeito Inibitório da via auditiva eferente; T1 = tipo 1 = monossomia do X; T2 = tipo 2 = outras alterações citogenéticas

Observou-se que o grupo estudo apresentou EIVE presente da OD significantemente menor que o grupo controle ( $p$-valor $=0,032)$, e não houve diferença significativa na supressão da $\mathrm{OE}$ entre os dois grupos.

A Tabela 3 apresenta o número (n) e a porcentagem dos resultados do EIVE (presente / ausente) segundo o cariótipo (T1 e T2).

Observou-se que não houve diferença significativa dos EIVE (presente/ausente) da OD e OE entre os dois grupos dos tipos de cariótipos.

\section{DISCUSSÃO}

Este estudo analisou o Efeito inibitório da via auditiva eferente em indivíduos com a ST e até onde sabemos, este é o primeiro estudo sobre o EIVE na ST. O GC apresentou valores estatisticamente mais elevados na OD, e no GE houve predominância do nível de resposta na OE. Esta condição atípica nos leva a acreditar em uma possível assimetria cortical relacionada com a produção e percepção da fala e outras habilidades neurocognitivas. Na ST as alterações neurocognitivas são variadas, e geralmente, são observadas dificuldades em habilidades não verbais, visuoespaciais/ perceptuais, memória, funções motoras, executivas e atencionais ${ }^{(16)}$ Apesar da predominância hemisférica central de um lado sobre o outro ser um tema muito estudado, é um desafio determinar os diversos níveis de predominância hemisférica para os sons verbais e não verbais, pois pode existir diferença na assimetria em um mesmo indivíduo ${ }^{(17)}$

Por outro lado, sabe-se que o grau de mosaicismo comumente varia entre os diferentes tipos de tecidos e órgãos, ainda assim em um estudo recente ao ser comparada a amostra de esfregaço bucal do lado direito e esquerdo, foi observada diferença no grau de mosaicismo entre os lados em metade da amostra avaliada com ST e o controle não apresentou variação entre os lados ${ }^{(18) .}$ 
Este achado pode estar relacionado com os dados encontrados em nosso estudo em que a resposta do EIVE foi mais elevada na $\mathrm{OE}$ dos participantes com ST, uma vez que além de saber da possibilidade de todas as pessoas com ST serem "mosaico" (19), os tecidos constituintes do SNC e da mucosa têm a mesma origem embriológica (20). Acreditamos que possa haver uma resposta para a lateralidade encontrada com base no que foi exposto acima.

Em relação à lateralidade do EIVE, ou seja, se existe diferença nos padrões de resposta do EIVE entre as orelhas direita e esquerda, sabe-se que existe vantagem da OD, o que reforça o conceito de lateralidade da função do sistema olivococlear, o que poderia indicar um atraso na condução sonora do trato olivococlear esquerdo em comparação com o direito ${ }^{(21)}$. Este dado pode ser observado em outros estudos que apontam maiores valores e ocorrência de EIVE na OD em indivíduos normoouvintes ${ }^{(22)}$. Além disso, outro estudo, também verificara a vantagem da orelha direita sobre a orelha esquerda apenas grupo controle e no grupo estudo a vantagem ocorreu na orelha esquerda ${ }^{(23) .}$ Em um estudo realizado, no qual comparou ratos pigmentados e albinos, observou-se diferenças na lateralidade dos neurônios do sistema auditivo eferente, fornecendo evidências de diferença no padrão de cruzamento da via olivococlear nesses animais (24).

Há controvérsias na literatura sobre o EIVE, que pode estar relacionado com a variabilidade do método empregado nos estudos, como: tipo do estímulo utilizado (Transiente ou produto de distorção); linear ou não-linear; a relação sinal/ruído considerada, utilização do índice bruto ou normalizador como critério de análise do resultado e as próprias diferenças inter sujeitos dentro de cada amostra. Os parâmetros aplicados no presente estudo foram os mais utilizados na literatura ${ }^{(14)}$ :estímulo Transiente, linear, à intensidade de $60 \mathrm{~dB}$, reprodutibilidade maior que $70 \%$, janela de análise de aproximadamente $20 \mathrm{~ms}$, faixa de frequência de $1 \mathrm{KHz}$ a $5 \mathrm{KHz}$. Foi considerada presença de resposta quando a amplitude das EOAT foi maior ou igual a 3 dB NPS acima do ruído, em no mínimo 3 frequências consecutivas. Este mesmo procedimento em seguida foi repetido, porém com a presença de ruído branco contralateral a $60 \mathrm{~dB}$ e utilizamos o índice bruto como critério de análise. O nível de ruído associado ao nível de resposta das EOA é um fator relevante para a quantificação da magnitude do EIVE. As variações no nível de resposta das EOA provenientes do ruído podem ser interpretadas como inibição fisiológica, ou seja, os baixos níveis de relação sinal/ruído das EOA pode dificultar

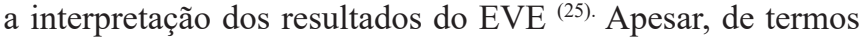
adotado em nosso protocolo de aplicação uma relação sinal ruído muito utilizada, porém considerada fraca, foi observada em toda a amostra estudada (GE e GC) relação sinal / ruído das EOA maior que $6 \mathrm{~dB}$, sendo este o mínimo desejável para detectar EIVE robusto e resultados repetíveis (26). Além disso, não houve variação significativa entre a relação sinal/ruído das EOA do grupo estudo e do grupo controle.

Ainda assim, os dados encontrados concordam com a literatura em que indivíduos normoouvintes e sem alterações podem apresentar lateralidade do EIVE na OD, entretanto é relevante que pesquisas futuras sejam realizadas comparando os critérios de aplicação e análise do EIVE para que sejam minimizadas tais controvérsias.

Não existe consenso na literatura sobre o padrão de normalidade do EIVE ${ }^{(27)}$, o que dificulta a comparação com estudos anteriores. No estudo onde foram comparados fumantes e não fumantes com idade entre 20 e 31 anos, o padrão de normalidade utilizado foi de $0,5 \mathrm{~dB}$ para caracterizar a presença do EIVE ${ }^{(27)}$. Já em outro estudo, no qual foi aplicado a pesquisa do EIVE em adultos (20 a 73 anos) de ambos os sexos, considerou-se presença de EIVE níveis de resposta acima de $0 \mathrm{~dB}^{(28)}$. No presente estudo adotamos como critério de normalidade EIVE maior ou igual a $1 \mathrm{~dB}$ por ser adotado em grande parte dos estudos ${ }^{(14 ; 15 ; 2)}$, e devido às características do equipamento utilizado. Ao observarmos a ocorrência do EIVE, em nosso estudo, a frequência foi baixa tanto no GE quanto no GC (Tabela 2), levantando a hipótese que o padrão de normalidade de $1 \mathrm{~dB}$ comumente utilizado, possa ser elevado, sugerindo a necessidade de mais estudos sobre o padrão de normalidade adotado para o EIVE ser considerado presente.

Ao pesquisarmos a possível relação do EIVE com a alteração citogenética da ST, não encontramos associação significativa nessa amostra em estudo, embora no grupo T1 (grupo com monossomia) tenha sido observada uma tendência clínica a apresentarem EIVE presente. Talvez estudos com um tamanho amostral maior possam verificar tal significância. Comparação com estudos anteriores não pode ser realizada, pois não foram encontrados estudos realizados sobre o tema.

Uma limitação do estudo foi o tamanho amostral. Dentre os indivíduos elegíveis, 35,39\% foram excluídos por apresentar audiometria alterada. Mesmo não sendo objetivo do nosso estudo, foi possível observar que todas as participantes com ST e audiometria normal (incluídas no GE) apresentaram resposta nas EOAT (M1), o que indica normalidade da função coclear. Tal achado é semelhante ao estudo realizado em pacientes com ST cuja média de idade foi inferior a 40 anos ${ }^{(29)}$. Entretanto, um estudo que avaliou 30 pacientes com ST cuja média de idade foi de 52 anos, verificou que $61,5 \%$ apresentaram alteração das EOAT contra apenas 46,2\% com alteração na audiometria. Este dado pode estar relacionado com a média de idade da amostra deste segundo estudo ser bem mais elevada do que o nosso. Ainda em relação às EOAT, observamos que não houve diferença significativa do nível de resposta (M1) entre o GE e o GC, tanto na OD quanto na OE. Esse achado provavelmente poderia diferir, se a média de idade da amostra do nosso estudo fosse maior, uma vez que indivíduos com ST podem apresentar presbiacusia precoce ${ }^{(4)} \mathrm{e}$, portanto, essa possível alteração coclear poderia impactar nas respostas das EOA. Devido à perda auditiva neurosensorial ser comumente associada à ST $(5 ; 6)$, a aplicação das EOAT como técnica para monitoramento da audição desses pacientes, poderia ser utilizada com o objetivo de detectar possíveis lesões cocleares precocemente, pensando que uma das funções das EOA é o monitoramento da função coclear, principalmente em pacientes com risco de perda auditiva. Esta identificação precoce pode ajudar no tratamento, antes mesmo, que ocorra alteração nos limiares audiométricos. ${ }^{(30)}$.

É importante ressaltar que o estudo inédito sobre a via auditiva eferente na ST provoca vários questionamentos, seja em relação à 
sua ocorrência, magnitude de respostas, lateralidade e associação com as diversas alterações citogenéticas na ST, demonstrando a necessidade de pesquisas futuras sobre o tema, com maior quantidade de participantes para obtermos conclusões mais consistentes.

\section{CONCLUSÃO}

No grupo de pacientes com ST o valor do EIVE foi significantemente maior na OE e significativamente menor que o grupo controle na OD. Não houve diferença significativa no EIVE da OD e da OE entre os dois tipos de cariótipo.

\section{AGRADECIMENTOS}

Agradeço a todos os voluntários que participaram da pesquisa e que contribuíram para a realização deste estudo.

\section{REFERÊNCIAS}

1. Kubba H, McAllister K, Hunter K, Mason A. Annual hearing screening in girls with Turner Syndrome: results from the first three years in Glasgow. Int J Pediatr Otorhinolaryngol. 2019;120:152-6. http://dx.doi.org/10.1016/j. ijporl.2019.02.025. PMid:30798112.

2. Mandelli AS, Abramides DVM. Manifestações clínicas e fonoaudiológicas na síndrome de Turner: estudo bibliográfico. Rev CEFAC. 2012;14(1):146-55.

3. Bois E, Nassar M, Zenaty D, Léger J, Abeele TVD, Teissier N. Otologic disorders in Turner syndrome. Eur Ann Otorhinolaryngol Head Neck Dis. 2018;135(1):21-4. http://dx.doi.org/10.1016/j.anorl.2017.08.006. PMid:28941966.

4. Bonnard Å, Bark R, Hederstierna C. Clinical update on sensorineural hearing loss in Turner syndrome and the X-chromossome. Am J Med Genet C Semin Med Genet. 2019;181(1):18-24. http://dx.doi.org/10.1002/ ajmg.c.31673. PMid:30632288.

5. Bazilio MM, Santos AFD, Almeida FG, Frota S, Guimarães M, Ribeiro MG. Association between cytogenetic alterationand the audiometric profile of individuals with Turner syndrome. Braz Journ Otorhinol. 2020; [In press].

6. Momensohn-Santos TM, Dias AMN, Valente CHB, Assayag FM. Prática da Audiologia Clínica. 6. ed. São Paulo: Editora Cortez; 2007. Anatomia e Fisiologia do órgão da Audição e do Equilíbrio; p. 12-43.

7. Fávero ML, Sanchez TG, Bento RF. Vias auditivas eferentes e seu papel no sistema auditivo. Arq Int Otorrinolaringol. 2001;72(5):575-9.

8. Guinan JJ Jr. Olivocochlear efferents: anatomy, physiology, function, and the measurement of efferent effects in humans. Ear Hear. 2006;27(6):589-607. http://dx.doi.org/10.1097/01.aud.0000240507.83072.e7. PMid:17086072.

9. Jesus NO, Angrisani RG, Maruta EC, Azevedo MF. Efeito de supressão das Emissões Otoacústicas em lactentes termo e pré-termo. CoDAS. 2016;28(4):3317. http://dx.doi.org/10.1590/2317-1782/20162015153. PMid:27509398.

10. Guinan JJ Jr, Backus BC, Lilaonitkul W, Aharonson V. Medial olivocochlear efferent reflex in humans: Otoacoustic Emission (OAE) measurement issues and the advantages of stimulus frequency OAEs. J Assoc Res Otolaryngol. 2003;4(4):521-40. http://dx.doi.org/10.1007/s10162-0023037-3. PMid:12799992.

11. OMS: Organização Mundial de Saúde [Internet]. Prevention of blindness and deafness: grades of hearing impairment. Geneve: WHO. 1997 [citado em 2017 Nov 04]. Disponível em: http://www.who.int/pdf/deafness/ hearing_impairment/grades/.

12. Hederstierna C, Hulcrantz M, Rosenhal U. Estrogen and hearing from a clinical point of view; characteristics of auditory function in Women with Turner syndrome. Hear Res. 2009;252(1-2):3-8. PMid:19095053.

13. Menegotto IH. Logoaudiometria básica. In: Bevilaqua F, Martinez S, Balen MAN, Pupo SA, Reis AC, Barbosa ACM, editores. Tratado de Audiologia. 1. ed. São Paulo: Santos; 2012, p. 81-99.
14. Durante AS. Emissões otoacústicas. In: Bevilaqua F, Martinez S, Balen MAN, Pupo SA, Reis AC, Barbosa ACM, editores. Tratado de Audiologia. 1. ed. São Paulo: Santos; 2012, p. 145-56.

15. Collet L, Veuillet E, Bene J, Morgan A. Effects of contralateral white noise on click - evoked emissions in normal and sensorineural ears: towards an exploration of the medial olivocochlear system. Audiology. 1992;31(1):1-7. http://dx.doi.org/10.3109/00206099209072897. PMid:1554329.

16. Antunes AM, Costa AJ, Haase VG. Variações cariotípicas na Síndrome de Turner: uma análise do fenótipo cognitivo. Rev Interinst Psic. 2015;8(2):34858.

17. Silva TR, Dias FAM. Laterality of activity of medial olivocochlear efferent system: prelinary study. Rev CEFAC. 2015;17(6):1855-62. http://dx.doi. org/10.1590/1982-021620151768615.

18. Thunström S, Landin-Wilhelmsen K, Bryman I, Hanson C. Side diferences in the degree of mosaicismo $f$ the buccal mucosa in Turner syndrome. Mol Genet Genomic Med. 2019;7(10):e00938. PMid:31466136.

19. Barros BA, Maciel-Guerra AT, Mello MP, Coeli FB, Carvalho AB, ViguettiCampos N, et al. A inclusão de novas técnicas de análise citogenética aperfeiçoou o diagnóstico cromossômico da síndrome de Turner. Arq Bras Endocrinol Metab. 2009;53(9):1137-42. http://dx.doi.org/10.1590/ S0004-27302009000900010.

20. Moore K, Persaud TVN, Tochia MG. A terceira semana do Desenvolvimento Humano. In: Moor K, editor. Embriologia Básica. 9. ed. London: Elsevier Health Sciences; 2016.

21. Ryan S, Kemp DT. The influence of evoking stimulus level on the neural suppression of transient evoked otoacoustic emissions. Hear Res. 1996;94(12):140-7. http://dx.doi.org/10.1016/0378-5955(96)00021-4. PMid:8789819.

22. Gkoritsa E, Korres S, Psarommatis I, Tsakanikos M, Apostolopoulos N, Ferekidis E. Maturation of the auditory system:1. Transient otoacoustic emissions as na index of inner ear maturation. Int J Audiol. 2007;46(6):2716. PMid: 17530511.

23. Veuillet E, Georgieff N, Philibert B, Dalery J, Marie-Cardine M, Collet L. Abnormal peripheral auditory asymmetry in schizophrenia. J Neurol Neurosurg Psychiatry. 2001;70(1):88-94. http://dx.doi.org/10.1136/ jnnp.70.1.88. PMid:11118254.

24. Reuss S, Closhen-Gabrisch S, Closhen C. The brainstem efferent acoustic chiasm in pigmented and albino rats. Hear Res. 2016;332:1-6. http://dx.doi. org/10.1016/j.heares.2015.11.012. PMid:26657095.

25. Mishra SK. Medial efferent mechanisms in children with auditory processing disorders. Front Hum Neurosci. 2014;8:860. http://dx.doi.org/10.3389/ fnhum.2014.00860. PMid:25386132.

26. Mishra SK, Lutman ME. Top-down influences of the medial olivocochlear efferent system in speech perception in noise. PLoS One. 2014;9(1):e85756. http://dx.doi.org/10.1371/journal.pone.0085756. PMid:24465686.

27. Paschoal CP, Azevedo MF. Cigarette smoking as a risk factor for auditory problems. Braz J Otorhinolaryngol. 2009;75(6):893-902. http://dx.doi. org/10.1016/S1808-8694(15)30556-5.

28. Oliveira M, Roberta J, Candido FF, Orozimbo ACF. Estudo da supressão da amplitude das Emissões Otoacústicas: dominância lateral. Brazilian Journal of Otorhinolaringology. 2011;77(5):547-54. http://dx.doi.org/10.1590/ S1808-86942011000500002.

29. Gawron W, Wikiera B, Rostkowska-Nadolska B, Orendorz-Fraczkowska K, Noczyńska A. Evaluation of hearing organ in patients with Turner syndrome. Int J Pediatr Otorhinolaryngol. 2008;72(5):575-9. http://dx.doi. org/10.1016/j.ijporl.2008.01.021. PMid:18343510.

30. Coelho MSB, Ferraz JRS, Almeida EOC, Almeida NA Fo. As emissões otoacústicas no diagnóstico diferencial das perdas auditivas induzidas por ruído. Rev CEFAC. 2010;12(6):1050-8. http://dx.doi.org/10.1590/S151618462010005000108 .

\section{Contribuição dos autores}

Todos os autores contribuíram para elaboração, confecção, coleta de dados, análise, escrita e lou supervisão das fases citadas. 